\title{
SIR epidemiological model with ratio-dependent incidence: influence of preventive vaccination and treatment control strategies on disease dynamics
}

\author{
Uday Kumar $^{1}$, PARTHA MANDAL ${ }^{1}$, Jai Tripathi², Sarita Bugalia², and Vijay Pal Bajiya ${ }^{2}$ \\ ${ }^{1}$ National Institute of Technology Patna \\ ${ }^{2}$ Central University of Rajasthan
}

January 17, 2021

\begin{abstract}
In this paper, we study an SIR epidemic model with ratio dependent incident rate function. We explore the impact of vaccination and treatment on the transmission dynamics of the disease. The treatment control strategies depend on the availability of maximal treatment capacity: treatment rate is constant when the number of infected individuals is greater than the maximal capacity of treatment and proportional to the number of infected individuals when the number of infected individuals is less than the maximal capacity of treatment. The existence and stability of the endemic equilibria are governed by the basic reproduction number and treatment control strategies. By carrying out rigorous mathematical analysis and numerical evaluations, it has been shown that (1) the sufficiently large value of the preventive vaccination rate can control the spread of disease, (2) a threshold level of the psychological (or inhibitory) effects in the incidence rate function is enough to decrease the infective population. Model system also undergoes a transcritical and a saddle-node bifurcation with respect to disease contact rate. In the presence of treatment strategies, system have multiple endemic equilibria and undergoes a backward bifurcation. The number of infected individuals decreases with respect to maximal treatment capacity and disease dies out from the system for large capacity of the treatment when constant treatment strategy is applied. Further, it is also found that the spread of disease can be suppressed by increasing treatment rate. Sensitivity analysis shows that the transmission and treatment rates are most sensitive parameters on the model system.
\end{abstract}

\section{Hosted file}

M2AS_SUBMITTED_191920.pdf available at https://authorea.com/users/389885/articles/504409sir-epidemiological-model-with-ratio-dependent-incidence-influence-of-preventivevaccination-and-treatment-control-strategies-on-disease-dynamics

\section{Hosted file}

M2AS_SUBMITTED_191920.tex available at https://authorea.com/users/389885/articles/504409sir-epidemiological-model-with-ratio-dependent-incidence-influence-of-preventivevaccination-and-treatment-control-strategies-on-disease-dynamics 
figures/fig2/fig2-eps-converted-to.pdf 
figures/EE1/EE1-eps-converted-to.pdf 
figures/fig1/fig1-eps-converted-to.pdf 
figures/fig3/fig3-eps-converted-to.pdf 
figures/fig4/fig4-eps-converted-to.pdf 
figures/fig5/fig5-eps-converted-to.pdf 
figures/I-k-K/I-k-K-eps-converted-to.pdf 
figures/I-K/I-K-eps-converted-to.pdf 
figures/I-r-rI-/I-r-rI--eps-converted-to.pdf 
figures/nonmon/nonmon-eps-converted-to.pdf 
figures/R-K/R-K-eps-converted-to.pdf 
figures/sn-1/sn-1-eps-converted-to.pdf 
figures/sn-2/sn-2-eps-converted-to.pdf 
figures/stability-1/stability-1-eps-converted-to.pdf 
figures/bif-R-0/bif-R-0-eps-converted-to.pdf 
figures/EE2/EE2-eps-converted-to.pdf 
figures/fig6/fig6-eps-converted-to.pdf 
figures/I-p-rI/I-p-rI-eps-converted-to.pdf 
figures/PRCC-K/PRCC-K-eps-converted-to.pdf 
figures/PRCC-RO/PRCC-R0-eps-converted-to.pdf 
figures/alpha-varyy-2/alpha-varyy-2-eps-converted-to.pdf 
figures/bifur-Ic-R-0-less-one/bifur-Ic-R-0-less-one-eps-converted-to.pdf 
figures/bilinear/bilinear-eps-converted-to.pdf 
figures/EE11/EE11-eps-converted-to.pdf 
figures/EE12/EE12-eps-converted-to.pdf 
figures/S-K/S-K-eps-converted-to.pdf 
figures/bifur-Ic-R-0-greater-one/bifur-Ic-R-0-greater-one-eps-converted-to.pdf 
figures/PRCC-RI/PRCC-RI-eps-converted-to.pdf 
figures/R-k-K/R-k-K-eps-converted-to.pdf 
figures/S-k-K/S-k-K-eps-converted-to.pdf 
figures/trans/trans-eps-converted-to.pdf 
figures/E02/E02-eps-converted-to.pdf 
figures/R-p-rI/R-p-rI-eps-converted-to.pdf 
figures/R-r-rI/R-r-rI-eps-converted-to.pdf 
figures/S-p-rI/S-p-rI-eps-converted-to.pdf 
figures/S-r-rI/S-r-rI-eps-converted-to.pdf 
figures/saturate/saturate-eps-converted-to.pdf 
figures/E01/E01-eps-converted-to.pdf 